\title{
Comparison of in vitro and in vivo phototoxicity tests with S-(-)-10,11-dihydroxyfarnesic acid methyl ester produced by Beauveria bassiana KACC46831
}

\author{
MIN-A KIM ${ }^{1 *}$, HYEONG-U SON $^{1 *}$, CHEOL-SIK YOON $^{2}$, SUNG-HEE NAM $^{3}$, \\ YOUNG-CHEOL CHOI ${ }^{3}$ and SANG-HAN LEE ${ }^{1,4}$
}

\author{
${ }^{1}$ Department of Food Science and Biotechnology, Graduate School, Kyungpook National University, Daegu 702-701; \\ ${ }^{2}$ Mycoplus Company Ltd., Anyang 431-080; ${ }^{3}$ Department of Agricultural Biology, National Academy of Agricultural Science, \\ Rural Development Agency, Suwon 441-707; ${ }^{4}$ Food and Bio-Industry Research Institute, \\ Kyungpook National University, Daegu 702-701, Republic of Korea
}

Received March 6, 2014; Accepted June 6, 2014

DOI: $10.3892 /$ br.2014.301

\begin{abstract}
Beauveria bassiana is a fungi that is well-known for demonstrating a resistance to environmental change. To confirm whether S-(-)-10,11-dihydroxyfarnesic acid methyl ester (DHFAME) produced by Beauveria bassiana KACC46831 causes phototoxicity when used for cosmetic purposes due to its anti-tyrosinase activity, we conducted in vitro and in vivo phototoxicity tests. There were no significant changes or damage observed in the compound-treated group with regards to skin phototoxicity, while 8-methoxypsoralen, which served as a positive control, induced toxic effects. The in vitro $3 \mathrm{~T} 3$ neutral red uptake assay, an alternative assessment, was used for further confirmation of the phototoxicity. The results showed that DHFAME did not exhibit phototoxicity at the designated concentrations, with or without UV irradiation in the 3T3 cells. These results indicated that the methyl ester produced by Beauveria bassiana KACC46831 does not induce phototoxicity in the skin. Therefore, the results of the present study indicate that DHFAME shows potential for use as a cosmetic ingredient that does not cause skin phototoxicity.
\end{abstract}

\section{Introduction}

Beauveria bassiana is well-known for its broad spectrum for hosts and has relative resistance to environmental

Correspondence to: Dr Sang-Han Lee, Department of Food Science and Biotechnology, Kyungpook National University, 1370 Sangyeok-dong, Buk-gu, Daegu 702-701, Republic of Korea

E-mail: sang@knu.ac.kr

*Conributed equally

Key words: Beauveria bassiana, S-(-)-10,11-dihydroxyfarnesic acid methyl ester, phototoxicity, 3T3 neutral red uptake test, cosmetic ingredient change (1). For centuries, adult Bombyx mori infected with Beauveria bassiana have been used as an oriental medicine for the treatment of stroke, hives and diabetes (2). To the best of our knowledge, Beauveria bassiana has a limited virulence in humans. Notably, only a few cases of invasive disease and keratitis have been documented, despite the widespread use of the organism (3). Entomopathogenic fungi, including Beauveria bassiana, Cordyceps sinensis, Cordyceps militaris, and Paecilomyces tenuipes, from a variety of resources have been employed for the treatment of atopic dermatitis, athlete's foot and dandruff (4). The use of this agent as a biological control has received increasing attention as Beauveria bassiana is used to exterminate a wide variety of pests $(1,5,6)$. The anti-bacterial activity of entomopathogenic fungi against food-borne bacterial growth has also been investigated (7).

Although these entomopathogenic fungi have been shown to possess valuable properties, including immune-modulation, anti-diabetic, anti-stress and antitumor activities (8), their application in the cosmetics industry has not been thoroughly studied. However, investigation into the whitening effects of fungal fermentation products has been performed (9), and the results of those studies indicated that phototoxicity tests are important for obtaining approval and authorization for the use of test compounds as functional cosmetic ingredients. Since there are numerous methods used to measure the toxicity of substances applied to the skin and skin-related tissues, various trials have been conducted to assess the biological effects of the cosmetic/cosmeceutical ingredients that are being approved (10). However, in vitro methods, such as the 3 T3 neutral red uptake (NRU) phototoxicity test (11) and local lymph node assay (12), are increasingly being used instead of animal models due to the ethical aspects involved. Emerging applications of insect extracts (or fractions) are employed to broaden the applicability of their biochemicals as cosmetics/cosmeceuticals. However, whether the agents produced by entomopathogenic fungi have adverse effects on exposed skin and eyes has yet to be determined. However, surplus reactions to cosmetics are frequent in patients with 
allergic contact dermatitis. A number of adverse outcomes, such as irritation, sensitization and acute/chronic toxicity, can be evaluated using in vitro, in vivo, semi-in vivo, and ex vivo animal models (13-15). The individual components or constituents should not exert toxic effects on the skin and should only be passed and approved in cases in which no eye lens damage/change is observed in animals or clinical trials for the development of cosmetics (16).

In the present study, the phototoxicity of S-(-)-10,11dihydroxyfarnesic acid methyl ester (DHFAME) was evaluated using an in vitro phototoxicity test and an in vivo animal model to determine whether the compound is safe for development in cosmetic applications.

\section{Materials and methods}

Chemicals. 8-Methoxypsoralen (8-MOP; M3501), polyethylene glycol (P3265), chloropromazine (CPZ; C0982), and neutral red (N4638) were purchased from Sigma-Aldrich Chemical Co., Ltd. (St. Louis, MO, USA). All media and compositions were commercially available.

Animal care and use. Seven-week-old Hartley guinea pigs, weighing 319.6-372.9 g, were purchased from Samtako Bio Korea (Osan, Korea) and used for the skin irritancy and phototoxicity tests, respectively. The animals were fed a commercial diet (Purina Korea, Inc., Seoul, Korea) and provided with water ad libitum throughout all the experiments. The study protocols complied with the guidelines of the International Association for the Study of Pain Committee for Research and Ethical Issues (17), and strictly adhered to the internal guidelines of the Kyungpook National University Animal Ethics Committee. All animals were acclimatized to the laboratory environment for $\sim 1$ week prior to commencement of the experiments. Five animals were allocated to each group.

Isolation and preparation of agent. DHFAME was produced by Beauveria bassiana KACC46831. Briefly, the fermentation medium consisted of $3 \%$ sucrose, $2 \%$ corn steep liquor, $0.05 \%$ potassium phosphate dibasic, $0.1 \%$ potassium phosphate monobasic and $0.05 \% \mathrm{MgSO}_{4} \cdot 6 \mathrm{H}_{2} \mathrm{O}$. The medium was prepared in a 5l-mini jar fermentor (Hankook Fermentor, Seoul, Korea) and sterilized at $121^{\circ} \mathrm{C}$ for $30 \mathrm{~min}$, subsequently it was chilled for inoculation of $5 \%$ culture. The fermentation was then carried out for 3 days, and subsequently the fermentation broth was centrifuged at $10.000 \mathrm{x}$ g for $10 \mathrm{~min}$ and the supernatant was added as previously described (18). The precipitate was then applied to an HP column chromatogram and high-performance liquid chromatography was performed with a reverse column (Waters, Milford, MA, USA) and a peak was obtained at a retention time of $7.662 \mathrm{~min}$ by a detector at $254 \mathrm{~nm}$ (2998 PDA; Waters). The peak was identified as S-(-)-10,11-dihydroxyfarnesic acid methyl ester by nuclear magnetic resonance and mass spectroscopy (18). A voucher specimen of the methyl ester produced by Beauveria bassiana KACC46831 has been deposited in the Laboratory of Food Enzyme Biotechnology, Kyungpook National University (Daego, Korea).

In vitro $3 T 3 \mathrm{NRU}$ test. The in vitro 3T3 NRU phototoxicity test was carried out as described previously (11) and by the
OECD guideline 432 (19). Briefly, 96-well plates (REF353072; BD Falcon, Franklin Lakes, NJ, USA) were seeded with $1.0 \times 10^{4}$ cells $/ \mathrm{ml}$ (total $100 \mu \mathrm{l}$ ) $3 \mathrm{~T} 3$ cells, and subsequently incubated at $37^{\circ} \mathrm{C}$ in a humidified $5 \% \mathrm{CO}_{2}$ incubator for $24 \mathrm{~h}$. Following the removal of the media and the washing of cells with Earle's balanced salt solution (EBSS), the cells were exposed to various dilutions (three replicate wells per concentration) of the test materials $(100 \mu \mathrm{l})$ in EBSS for $60 \mathrm{~min}$. The cells were treated with an initial range of nine concentrations ranging from 0 to $100 \mu \mathrm{M} \mathrm{CPZ}$ (as a positive control) or 0 to $250 \mu \mathrm{M}$ DHFAME. Following incubation for $24 \mathrm{~h}$ in a $\mathrm{CO}_{2}$ incubator at $37^{\circ} \mathrm{C}$, duplicate plates were either exposed to UVA/visible light at $5 \mathrm{~J} / \mathrm{cm}^{2}$ (LF-206.LS; UVitec Strasbourg, France) or kept in the dark for $50 \mathrm{~min}$. Following irradiation, the media were discarded from all the plates and the cells were washed with culture medium. The cells were then reincubated in culture medium overnight. On day 3 , the medium was removed and the cells were washed with pre-warmed buffer and added to $100 \mu \mathrm{l}$ of neutral red medium $(50 \mu \mathrm{g} / \mathrm{ml}$, serum-free $)$. Samples were then incubated for $3 \mathrm{~h}$ in a $\mathrm{CO}_{2}$ incubator at $37^{\circ} \mathrm{C}$, and subsequently $150 \mathrm{ml}$ of neutral red extraction solution (distilled water:ethyl alcohol:acetone $=49: 50: 1)$ was added to the plates. The plates were then agitated and the optical density was measured at $540 \mathrm{~nm}$ using a spectrophotometer (Perkin Elmer Wallac, Inc., Turku, Finland).

In vivo phototoxicity test. An in vivo phototoxicity test was conducted using Hartley guinea pigs. The animals were divided into an untreated, three experimental $(10,30$ and $100 \mathrm{mg} / \mathrm{ml}$ of DHFAME) and a positive control group that was treated with 8-MOP. Each group contained five guinea pigs (7-week-old males, weighing 319.6-372.9 g). The untreated group was exposed to polyethylene glycol. For the three experimental groups, $0.5 \mathrm{ml} / \mathrm{site}$ of the solution was applied. The treated skin was then irradiated with UV light at a distance of $10 \mathrm{~cm}$ for 10 min using UV irradiation apparatus (UVITEC LF-206.LS) with a UV lamp (365 nm). The left site was designated as the light irradiation site, whereas the right site was not irradiated. After 2, 4 and $24 \mathrm{~h}$ of irradiation, any skin erythema, eschar and swelling was scored relative to the control. Transdermal administration was carried out by removing the fur in a $4 \times 6 \mathrm{~cm}^{2}$ area with an electric hair cutter and then applying the test sample to two regions (each $2 \times 2 \mathrm{~cm}^{2}$ ). The test groups were treated with $0.5 \mathrm{ml}$ of DHFAME at concentrations of 10,30 and $100 \mathrm{mg} / \mathrm{ml}$, whereas $0.5 \mathrm{ml}$ of a $0.1 \%$ 8-MOP solution was applied to each side of the test site as a positive control (20). The non-irradiation site was shielded by aluminum tape.

Statistical analysis. Data are presented as the means \pm standard deviation. Statistical analysis was carried out by Probit analysis using the SPSS 9.0 program (SPSS, Inc., Chicago, IL, USA). $\mathrm{P}<0.05$ was considered to indicate a statistically significant different following analysis using Pearson's goodness-of-fit test.

\section{Results and Discussion}

Throughout the evaluation of active components that exhibit whitening activities for application as a cosmetic from natural 
Table I. Comparison of the phototoxicity test evaluating the effects of S-(-)-10,11-dihydroxyfarnesic acid methyl ester (DHFAME) produced by Beauveria bassiana KACC46831.

\begin{tabular}{|c|c|c|c|c|c|c|}
\hline \multirow[b]{2}{*}{ Criteria } & \multirow[b]{2}{*}{ Total scores } & \multirow[b]{2}{*}{ Distilled water } & \multicolumn{3}{|c|}{ DHFAME, mg/ml } & \multirow[b]{2}{*}{$0.1 \% 8-\mathrm{MOP}$} \\
\hline & & & 10 & 30 & 100 & \\
\hline Non-irritating & $0.0-0.5$ & Yes & Yes & Yes & Yes & \\
\hline Minimally irritating & $0.6-1.2$ & & & & & \\
\hline Obviously irritating & $1.3-2.5$ & & & & & Yes \\
\hline Extremely irritating & $2.6-5.0$ & & & & & \\
\hline
\end{tabular}

8-MOP, 8-methoxypsoralen.

resources, Beauveria bassiana KACC46831 was found to produce a potent compound during liquid culture. The compound was identified as DHFAME and found to exert anti-tyrosinase activity in vitro and in vivo [(12) and data not shown].

In a previous study, we examined whether the agent had the ability to ameliorate skin inflammation, including atopic dermatitis (18). Initially, insect biomaterials were obtained and processed into biomaterials using a variety of methods. Subsequently, microbial fermentation, biotransformation, supercritical extraction or chemical modification techniques were employed to convert the raw extracts into a cosmetic, cosmeceutical, neutraceutical or hit/lead drug. Therefore, the development of anti-tyrosinase agents from medicinal insect extracts was tested, which revealed that the methyl ester had potent whitening activity (18). To determine the toxicity of the agent, an acute toxicity test was conducted for the application of cosmetic ingredients.

3T3 NRU phototoxicity was first tested in vitro according to the OECD 432 guideline. For the assay, CPZ was selected as a positive control, as the OECD guideline suggests that this drug exhibits phototoxicity by UV irradiation in 3T3 cells. As shown in Fig. 1A, 3T3 cells showed characteristic features of growth in the presence of various concentrations of CPZ without UV in a concentration-dependent manner. In particular, 50 and $100 \mu \mathrm{M} \mathrm{CPZ}$ exhibited 88 and $21.5 \%$ cell viability, respectively, when compared to the control (Fig. 1; dotted and straight lines). When the cells were treated with $\mathrm{UV}$ and $10 \mu \mathrm{M} \mathrm{CPZ}$, the growth was decreased significantly by $<37.4 \%$ (Fig. 1A). Cell viability was $0 \%$ in response to treatment with $15 \mu \mathrm{M} \mathrm{CPZ}$ with UV (Fig. 1B; comparison of upper and lower panels). The probable toxicity rate of $\mathrm{CPZ}$ was 1.000, whereas the rates of PIF and MPE were 12.016 and 0.781 , respectively. This finding suggested that CPZ treatment results in phototoxicity to UV irradiation. Under these conditions, various concentrations of DHFAME were compared to the positive control. As shown in Fig. 1C, a higher concentration of DHFAME did not cause a notable decrease in cell viability with or without UV (dotted and straight lines, respectively) at $<250 \mu \mathrm{M}$. Moreover, the cell morphology did not change unexpectedly at the designated concentration (Fig. 1D). The phototoxicity irritancy factor (PIF) and mean photo effect (MPE) of CPZ was 12.016 and 0.781, respectively, indicating that the probable phototoxicity rate was 1.000 and that DHFAME did not induce phototoxicity in this in vitro $3 \mathrm{~T} 3$ NRU phototoxicity test (data not shown). Conversely, the PIF of DHFAME was $<1.000$ and the MPE was 0.060, indicating that the probable phototoxicity rate was 0.003 (data not shown).

To determine whether DHFAME exhibited phototoxicity in vivo, DHFAME produced by Beauveria bassiana KACC46831 was soaked on the skin of guinea pigs and the toxicity was determined compared to guinea pigs treated with 8-MOP. The lesions were examined at 2, 4 and $24 \mathrm{~h}$ after application of DHFAME to evaluate phototoxicity. In particular, erythema and eschar were determined by observation with the naked eye using the following scale: 0 , no erythema; 1 , extremely slight; 2 , well-defined; 3 , moderate to severe; and 4 , severe erythema to slight eschar formation.

Phototoxicity was subsequently evaluated by analyzing the skin exposed to UV irradiation. Following fur removal, guinea pig skin was treated with DHFAME and 8-MOP, and the degree of erythema was determined using the aforementioned scale. For up to $4 \mathrm{~h}$ after UV irradiation, similar erythema symptoms were observed. After $24 \mathrm{~h}$, the DHFAME-treated groups showed no symptoms of toxicity in the skin, whereas the 8-MOP group ( $0.1 \%$ as a positive control) showed moderate to severe erythema (Fig. 2). To measure edema, the following scale was used: 0 , no edema; 1 , extremely slight; 2 , well-defined; 3 , moderate to severe; and 4, severe edema. The results showed that DHFAME did not cause erythema or eschar, whereas 8-MOP resulted in slight edema (Fig. 2). A final score was then determined by assessing the total scores for erythema, edema and crust as follows: 0.0-0.5, almost no phototoxic resistance; 0.6-1.2, weakly phototoxic; 1.3-2.5, clearly and highly phototoxic; and 2.6-5.0, highly and severely phototoxic. As shown in Table I, the three samples $(10,30$ and $100 \mathrm{mg} / \mathrm{ml})$ were associated with scores of only $0.0-0.5$, suggesting that the agent tested in the experiment was non-irritating. However, treatment with 8-MOP was a clearly irritating compound that resulted in erythema, eschar, and edema (Fig. 2). After 2 to $4 \mathrm{~h}$ of UV irradiation, a slight redness was observed in all agent-treated groups, but this redness disappeared after $24 \mathrm{~h}$. Conversely, the groups treated with 8-MOP developed erythema and edema, indicating that the overall condition of the phototoxicity test was achieved. Taken together, these findings indicate that 8-MOP treatment induced erythema, edema, and/or eschar in a concentration-dependent manner, whereas DHFAME had no effect. 

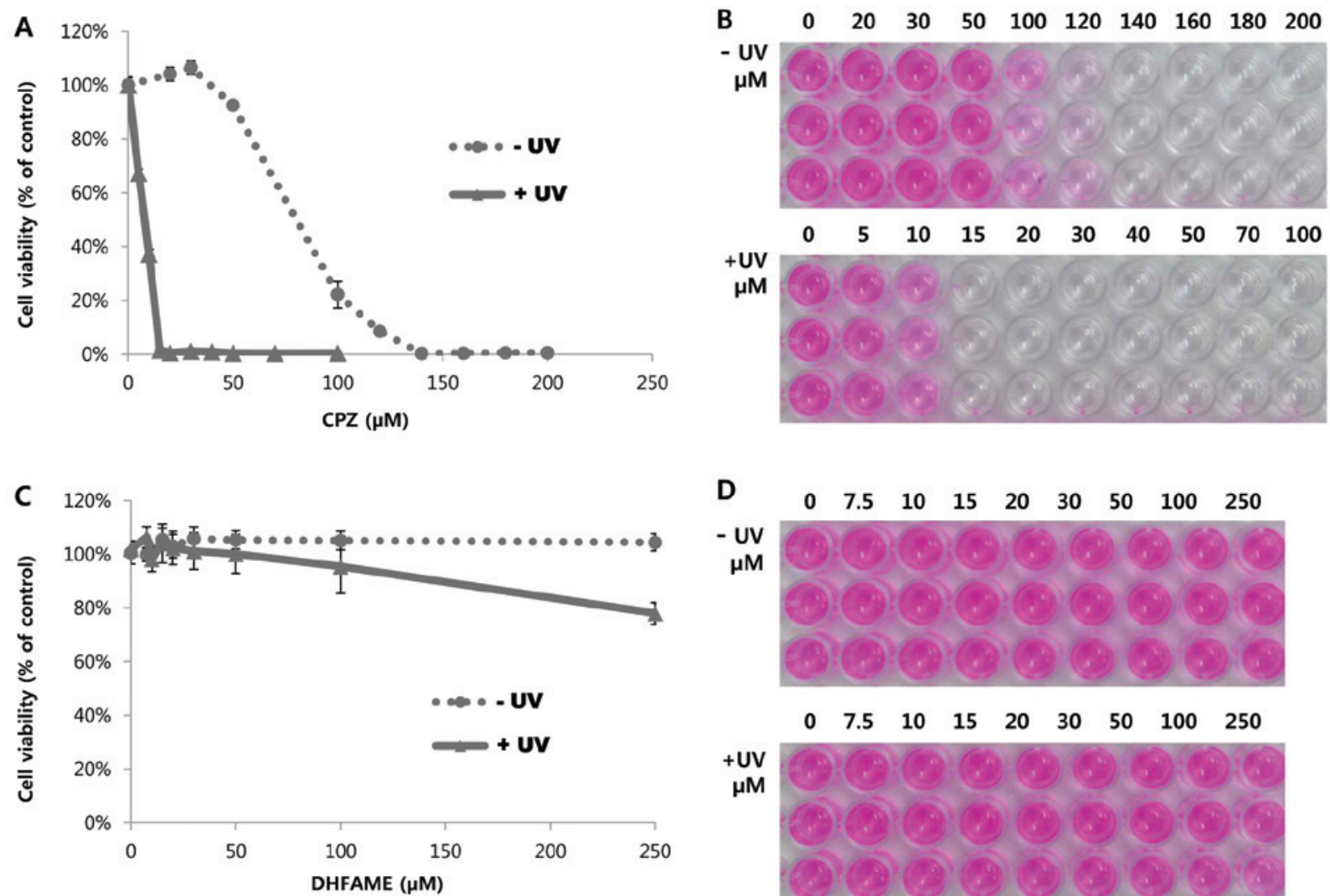

D
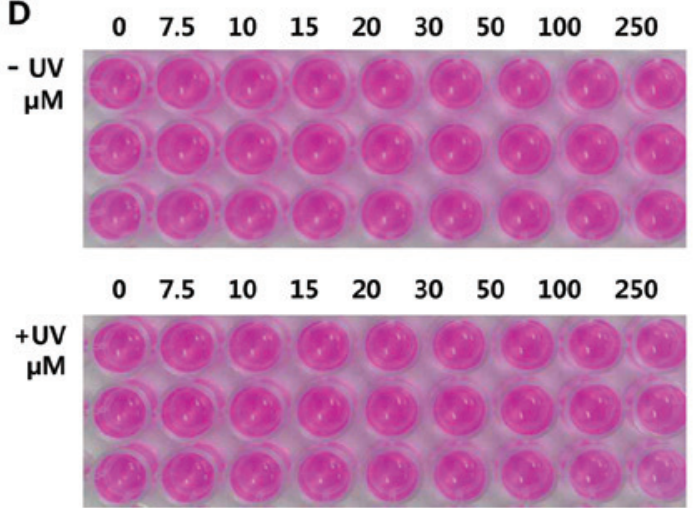

Figure 1. In vitro 3T3 neutral red uptake (NRU) phototoxicity tests of S-(-)-10,11-dihydroxyfarnesic acid methyl ester (DHFAME) produced by Beauveria bassiana KACC46831. (A and C) The in vitro 3T3 NRU phototoxicity test was carried out and characteristic features of growth in the presence of various concentrations of CPZ without UV were identified in a concentration-dependent manner (dotted and straight lines). (B) The cells were killed by UV irradiation with CPZ, (D) whereas the cells remained alive following UV irradiation with DHFAME.

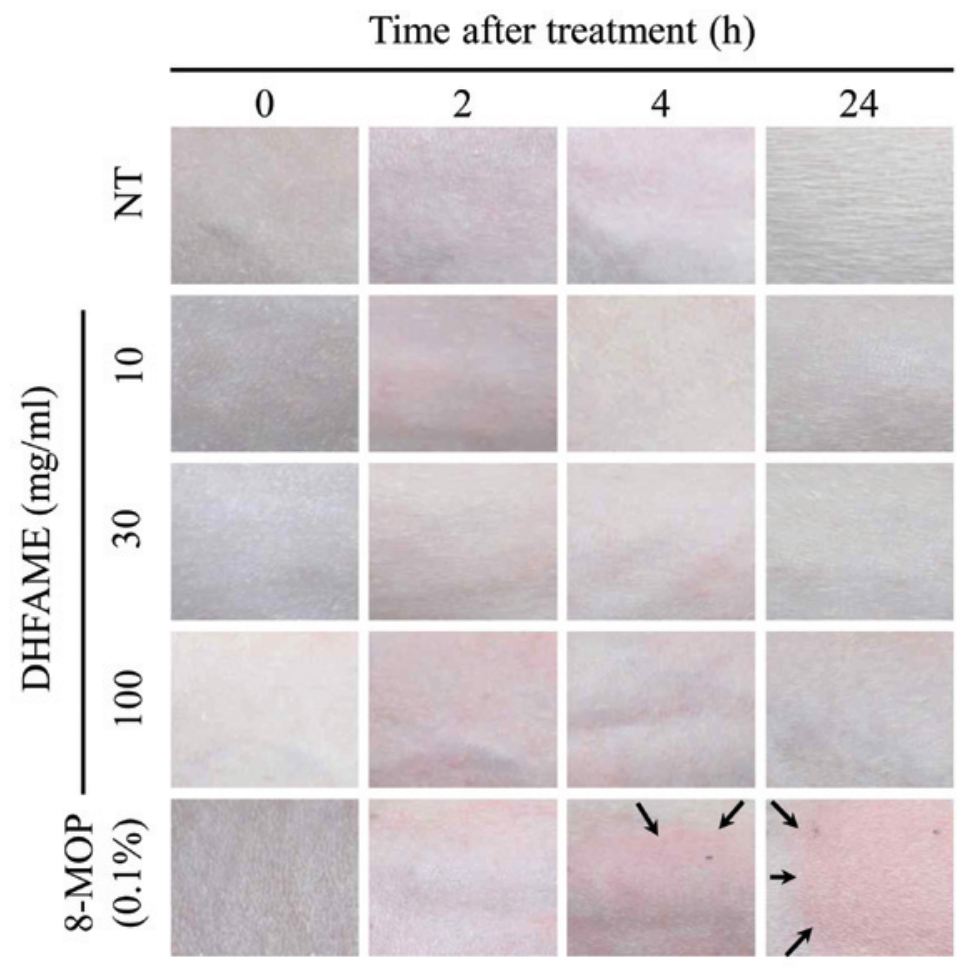

Figure 2. In vivo phototoxicity tests of S-(-)-10,11-dihydroxyfarnesic acid methyl ester (DHFAME) produced by Beauveria bassiana KACC46831. The images show the shaved backs of guinea pigs. The positive control group treated with 8-MOP showed a toxic response at $2 \mathrm{~h}$ after $\mathrm{UV}$ irritation, and gradually degenerated. The arrows indicate swelling and erythema with phototoxicity and the dotted boxes show that 8-methoxypsoralen (8-MOP) was applied to the area. Data are a classical set of five independent experiments. 
In summary, the present study investigated whether DHFAME has the potential to cause skin phototoxicity. None of the investigated concentrations of DHFAME were found to irritate the skin or were phototoxic, indicating that DHFAME may be useful in the cosmetic or cosmeceutical industry and for other applications. Although DHFAME was derived from an entomopathogenic fungus, its potential mode of action and toxicity require further evaluation.

\section{Acknowledgements}

The present study was supported by the Bio-Green21 Agenda Project (grant no. PJ009608012013). The authors would like to thank Mr. Dong-Yoon Nam and Mr. Yong-Soo Cha for their technical assistance.

\section{References}

1. Ownley BH, Griffin MR, Klingeman WE, Gwinn KD, Moulton JK and Pereira RM: Beauveria bassiana: endophytic colonization and plant disease control. J Invertebr Pathol 98: 267-270, 2008

2. Pemberton RW: Insects and other arthropods used as drugs in Korean traditional medicine. J Ethnopharmacol 65: 207-216, 1999.

3. Figueira L, Pinheiro D, Moreira R, Pinto E, Simões J, Camisa E, Torrão L, Palmares J and Falcão-Reis F: Beauveria bassiana keratitis in bullous keratopathy: antifungal sensitivity testing and management. Eur J Ophthalmol 22: 814-818, 2012.

4. Zhou X, Gong Z, Su Y, Lin J and Tang K: Cordyceps fungi: natural products, pharmacological functions and developmental products. J Pharm Pharmacol 61: 279-291, 2009.

5. Fernandes ÉK, Bittencourt VR and Roberts DW: Perspectives on the potential of entomopathogenic fungi in biological control of ticks. Exp Parasitol 130: 300-305, 2012.

6. Madsen AM: Occupational exposure to microorganisms used as biocontrol agents in plant production. Front Biosci (Schol Ed) 3: 606-620, 2011.

7. Seo ST, Lee JS, Park JH, Han KS and Jang HI: Investigation of antibiotic susceptibility of some plant pathogenic bacteria. Kor J Food Sci Technol 23: 495-498, 2005.

8. Wang Q and Xu L: Beauvericin, a bioactive compound produced by fungi: a short review. Molecules 17: 2367-2377, 2012.
9. Nam SH, Yoon CS, Jeon JY, Lee SH, Lee KG, Yeo JH and Hwang JS: Composition exhibiting melanin-inhibiting activity. Republic of Korea KR Patent 10-1239631. Filed March 28, 2011; issued Feb 27, 2013.

10. Nigam PK: Adverse reactions to cosmetics and methods of testing. Indian J Dermatol Venereol Leprol 75: 10-18, 2009.

11. Clothier RH: Phototoxicity and acute toxicity studies conducted by the FRAME Alternatives Laboratory: a brief review. Altern Lab Anim 35: 515-519, 2007.

12. Goebel C, Aeby P, Ade N, Alépée N, Aptula A, Araki D, Dufour E, Gilmour N, Hibatallah J, Keller D, Kern P, Kirst A, Marrec-Fairley M, Maxwell G, Rowland J, Safford B, Schellauf F, Schepky A, Seaman C, Teichert T, Tessier N, Teissier S, Weltzien HU, Winkler P and Scheel J: Guiding principles for the implementation of non-animal safety assessment approaches for cosmetics: skin sensitisation. Regul Toxicol Pharmacol 63: 40-52, 2012.

13. Tavaszi J, Budai P, Pálovics A and Kismányoki A: An alternative test battery in detecting ocular irritancy of agrochemicals. Commun Agric Appl Biol Sci 73: 891-895, 2008.

14. Scott L, Eskes C, Hoffmann S, et al: A proposed eye irritation testing strategy to reduce and replace in vivo studies using Bottom-Up and Top-Down approaches. Toxicol In Vitro 24: 1-9, 2010.

15. Osborne R, Perkins MA and Roberts DA: Development and intralaboratory evaluation of an in vitro human cell-based test to aid ocular irritancy assessments. Fundam Appl Toxicol 28: 139-153, 1995.

16. Nolan KA and Marmur ES: Over-the-counter topical skincare products: a review of the literature. J Drugs Dermatol 11: 220-224, 2012

17. Zimmermann M: Ethical guidelines for investigations of experimental pain in conscious animals. Pain 16: 109-110, 1983.

18. Nam SH, Yoon CS and Lee SH: Final report of development on bioactive compounds derived from entomopathogenic fungi. In: Rural Development Agency of Korea, pp1-100, 2011 (http://lib. rda.go.kr/newlib).

19. Peters B and Holzhütter HG: In vitro phototoxicity testing: development and validation of a new concentration response analysis software and biostatistical analyses related to the use of various prediction models. Altern Lab Anim 30: 415-432, 2002.

20. Neumann NJ, Blotz A, Wasinska-Kempka G, Rosenbruch M, Lehmann P, Ahr HJ and Vohr HW: Evaluation of phototoxic and photoallergic potentials of 13 compounds by different in vitro and in vivo methods. J Photochem Photobiol B 79: 25-34, 2005. 